\title{
NEMA NU 2-2007 performance characteristics of GE Signa integrated PET/ MR for different PET isotopes
}

\author{
Paulo R. R. V. Caribé ${ }^{1,2^{*}}$ (D, M. Koole ${ }^{2}$, Yves D'Asseler ${ }^{1}$, Timothy W. Deller ${ }^{3}$, K. Van Laere ${ }^{2}$ and S. Vandenberghe ${ }^{1}$
}

\author{
* Correspondence: paulo.caribe@ \\ ugent.be \\ ${ }^{1}$ Medical Imaging and Signal \\ Processing - MEDISIP, Ghent \\ University, Corneel Heymanslaan 10, \\ 9000 Ghent, Belgium \\ ${ }^{2}$ Division of Nuclear Medicine and \\ Molecular Imaging, UZ/KU Leuven, \\ Herestraat 49 B-3000, Leuven, \\ Belgium \\ Full list of author information is \\ available at the end of the article
}

\begin{abstract}
Background: Fully integrated PET/MR systems are being used frequently in clinical research and routine. National Electrical Manufacturers Association (NEMA) characterization of these systems is generally done with ${ }^{18} \mathrm{~F}$ which is clinically the most relevant PET isotope. However, other PET isotopes, such as ${ }^{68} \mathrm{Ga}$ and ${ }^{90} \mathrm{Y}$, are gaining clinical importance as they are of specific interest for oncological applications and for follow-up of ${ }^{90} \mathrm{Y}$-based radionuclide therapy. These isotopes have a complex decay scheme with a variety of prompt gammas in coincidence. ${ }^{68} \mathrm{Ga}$ and ${ }^{90} \mathrm{Y}$ have higher positron energy and, because of the larger positron range, there may be interference with the magnetic field of the MR compared to ${ }^{18} \mathrm{~F}$. Therefore, it is relevant to determine the performance of PET/MR for these clinically relevant and commercially available isotopes.
\end{abstract}

Methods: NEMA NU 2-2007 performance measurements were performed for characterizing the spatial resolution, sensitivity, image quality, and the accuracy of attenuation and scatter corrections for ${ }^{18} \mathrm{~F},{ }^{68} \mathrm{Ga}$, and ${ }^{90} \mathrm{Y}$. Scatter fraction and noise equivalent count rate (NECR) tests were performed using ${ }^{18} \mathrm{~F}$ and ${ }^{68} \mathrm{Ga}$. All phantom data were acquired on the GE Signa integrated PET/MR system, installed in UZ Leuven, Belgium.

Results: ${ }^{18} \mathrm{~F},{ }^{68} \mathrm{Ga}$, and ${ }^{90} \mathrm{Y}$ NEMA performance tests resulted in substantially different system characteristics. In comparison with ${ }^{18} \mathrm{~F}$, the spatial resolution is about $1 \mathrm{~mm}$ larger in the axial direction for ${ }^{68} \mathrm{Ga}$ and no significative effect was found for ${ }^{90} \mathrm{Y}$. The impact of this lower resolution is also visible in the recovery coefficients of the smallest spheres of ${ }^{68} \mathrm{Ga}$ in image quality measurements, where clearly lower values are obtained. For ${ }^{90} \mathrm{Y}$, the low number of counts leads to a large variability in the image quality measurements. The primary factor for the sensitivity change is the scale factor related to the positron emission fraction. There is also an impact on the peak NECR, which is lower for ${ }^{68} \mathrm{Ga}$ than for ${ }^{18} \mathrm{~F}$ and appears at higher activities.

Conclusions: The system performance of GE Signa integrated PET/MR was substantially different, in terms of NEMA spatial resolution, image quality, and NECR for ${ }^{68} \mathrm{Ga}$ and ${ }^{90} \mathrm{Y}$ compared to ${ }^{18} \mathrm{~F}$. But these differences are compensated by the PET/MR scanner technologies and reconstructions methods.

Keywords: PET/MR, NEMA NU 2-2007, ${ }^{18} \mathrm{~F}^{68}{ }^{6} \mathrm{Ga},{ }^{90} \mathrm{Y}$

\section{Background}

The Signa PET/MR has MR-compatible silicon photomultiplier (SiPM) detector technology characterized by a superior light detection as compared to conventional PET

(c) The Author(s). 2019 Open Access This article is distributed under the terms of the Creative Commons Attribution 4.0 International License (http://creativecommons.org/licenses/by/4.0/), which permits unrestricted use, distribution, and reproduction in any medium, provided you give appropriate credit to the original author(s) and the source, provide a link to the Creative Commons license, and indicate if changes were made. 
technology [1-3]. The advantage of SiPMs versus avalanche photodiodes (APDs) is a faster response, enabling the combination of excellent time-of-flight (TOF) PET (close to $400 \mathrm{ps}$ ) imaging with MR scanning. The smaller detector bore and long axial extent $(25 \mathrm{~cm})$ of the PET ring (in comparison to state-of-the-art PET/CT) result in a superior sensitivity of $21 \mathrm{cps} / \mathrm{kBq}$, thus allowing a lower PET tracer dosing besides the evident dose reduction by omitting the CT [4]. Hybrid PET/MR is a relatively new multimodality imaging technique and offers the potential for combined structural, functional, and molecular imaging assessment of a wide variety of oncologic, neurologic, cardiovascular, and musculoskeletal conditions $[5,6]$. However, the challenges beyond those of a technical nature remain for PET/MR imaging, including the standardization of appropriateness criteria, image acquisition parameters, and clinically relevant and as well commercially available isotopes.

With PET becoming more widely used, the transport logistics have allowed faster shipments of radioisotopes to small imaging centers. The majority of PET studies in clinical routine are still being performed with ${ }^{18} \mathrm{~F}$, because of its physical properties combined with efficient transportation logistics which widely increase its availability. The same holds for ${ }^{68} \mathrm{Ga}$ and ${ }^{90} \mathrm{Y}$, of which the use is not dependent on the availability of a cyclotron. However, the physical properties of the PET radioisotopes are quite different from ${ }^{18} \mathrm{~F} .{ }^{18} \mathrm{~F}$ almost exclusively decays via positron emission (96.8\%) and with a relatively low maximum energy of the positron of $0.6335 \mathrm{MeV}$. The maximum and mean range of ${ }^{18} \mathrm{~F}$ are equal to 2.4 and $0.6 \mathrm{~mm}$. The other $3 \%$ of decays is via electron capture [7].

The use of the generator-based isotope ${ }^{68} \mathrm{Ga}$ has seen a steady increase in the last years. It is obtained from a ${ }^{68} \mathrm{Ge} /{ }^{68} \mathrm{Ga}$ generator obviating the need for a cyclotron on site. One generator will typically be used for about 1 year [8] and the equilibrium between ${ }^{68} \mathrm{Ga}$ and ${ }^{68} \mathrm{Ge}$ is re-attained rapidly enough to allow multiple radiotracers preparations a day. ${ }^{68} \mathrm{Ga}$ is used for labeling both small compound and macromolecules, such as ${ }^{68} \mathrm{Ga}$-PSMA targeting the prostate-specific membrane antigen or ${ }^{68} \mathrm{Ga}$-labeled tracers targeting the somatostatin receptor expressed by neuroendocrine tumors, which are considered as key applications for combined PET/MR [9-11]. ${ }^{68} \mathrm{Ga}$ is not a pure positron emitter and has a more complex decay scheme than ${ }^{18} \mathrm{~F}$. Non-pure isotopes emit additional gammas that may even directly fall into the energy window accepted by the PET scanner. These high-energy gammas have some probability of generating spurious coincidences after scattering in the patient or via $e^{+} / e^{-}$pair production in the detector or the patient [12-14]. In $87.8 \%$ of the decays, ${ }^{68} \mathrm{Ga}$ will emit a positron with a maximum energy of $1.899 \mathrm{MeV}$ and a mean energy of $0.89 \mathrm{MeV}$ with a half-life $t_{1 / 2}=$ $67.6 \mathrm{~min}$. The much higher energy of the positron emission (compared to ${ }^{18} \mathrm{~F}$ ) leads to an increased maximum and mean range of 8.9 and $2.9 \mathrm{~mm}$. It also emits additional gammas of $578.52 \mathrm{keV}$ (0.034), $805.83 \mathrm{keV}$ (0.094), $1077.35 \mathrm{keV}(3.22), 1261.08 \mathrm{keV}$ (0.094), and $1883.16 \mathrm{keV}(0.137)$.

In the same way, ${ }^{90} \mathrm{Y}$ has rapidly gained attention as one of the most widely used therapeutic radioisotope in nuclear medicine. ${ }^{90} \mathrm{Y}$ is used in radioembolization of liver tumors. Tiny glass or resin beads called microspheres are administered in the hepatic artery and are transported into the blood vessels at the tumor site. The spheres get physically trapped and the radioactive isotope ${ }^{90} \mathrm{Y}$ delivers a high dose (via electrons) of radiation to the tumor. Several centers also use their PET system to image the therapeutic isotope ${ }^{90} \mathrm{Y}$. Studies have shown that ${ }^{90}$ Y-DOTA and ${ }^{90}$ Y-DTPA have potential in 
intra-vascular radionuclide therapy and ${ }^{90} \mathrm{Y}$ can simultaneously work as an imaging agent and a therapeutic [15-17]. ${ }^{90} \mathrm{Y}$ is mainly a $\beta^{-}$emitter with a very small branching ratio for positron production. In $0.003186 \%$ of the decays, there will be the emission of an $e^{+} / e^{-}$pair at $1.76 \mathrm{MeV}$. As the transition energy is $1.76 \mathrm{MeV}$, it remains $738 \mathrm{keV}$ kinetic energy to be split between the electron and the positron in order to conserve the null momentum. With a half-life of $64.1 \mathrm{~h},{ }^{90} \mathrm{Y}$ produces a weak but useable PET signal $[7,18]$, as illustrated by several clinical and phantom studies.

Furthermore, these isotopes may be of particular interest for PET/MR in prostate cancer, liver studies, and follow-up of radionuclide therapy. Therefore, it is relevant to determine the performance of PET/MR for these clinically relevant and commercially available isotopes in order to ensure correct functionality and optimal image quality. For PET scanners in particular, the National Electrical Manufacturers Association (NEMA) has defined a standard to assess the performance of the tomographic system, which is widely accepted by manufacturers [19]. The NEMA NU 2-2007 standard identifies ${ }^{18} \mathrm{~F}$ as the radionuclide to be used for all tests. Due to factors such as positron range, interference with magnetic field, and non-pure emissions with additional gammas, the results may be different when nonconventional radioisotopes, such as ${ }^{68} \mathrm{Ga}$ and ${ }^{90} \mathrm{Y}$ [20-22], would be used. The aim of this study is to assess the impact of using different PET isotopes for the NEMA tests performance evaluation of the GE Signa integrated PET/MR. NEMA NU 2-2007 performance measurements for characterizing spatial resolution, sensitivity, image quality, accuracy of attenuation and scatter corrections (IQ), and noise equivalent count rate (NECR) were performed using ${ }^{18} \mathrm{~F}$ and ${ }^{68} \mathrm{Ga}$. For ${ }^{90} \mathrm{Y}$, all tests except NECR tests were also performed.

\section{Methods}

All phantom experiments were performed on the MP24 version of the GE Signa integrated PET/MR whole-body hybrid system, installed in UZ Leuven, Belgium. The MR component of the hybrid system consists of a 3.0 Tesla static magnetic field, a radiofrequency (RF) transmit body coil, and a gradient coil system which provide a maximum amplitude of 44 $\mathrm{mT} / \mathrm{m}$ and a maximum slew rate of $200 \mathrm{~T} / \mathrm{m} / \mathrm{s}$. The PET component is comprised of 5 detector rings, each consisting of 28 detector blocks. Total axial FOV is equal to $25 \mathrm{~cm}$. Table 1 contains a summary of important design and performance parameters.

The PET detectors are based on a lutetium-based scintillator (LYSO) readout with MR-compatible silicon photomultiplier technology [3]. Before NEMA testing, a well counter calibration scan was performed with ${ }^{18} \mathrm{~F}$ in a uniform cylindric phantom. As a recommended calibration, the activity injected was measured using two dose calibrators (Capitec-CRC-55tR) with settings for different isotopes. The following measurements were performed according to the NEMA NU 2-2007 protocol.

\section{Spatial resolution}

A high activity concentration of approximately $200 \mathrm{MBq} / \mathrm{ml}$ was used to generate point sources (drop of activity raised in a capillary). In total, more than 500,000 counts were acquired. Both axial and transaxial resolution were measured at two different positions in the axial $z$-direction: in the central position of the FOV and at a position a quarter of the total axial FOV away from the center. The source point position was adjusted until all $x-y-z$ values fall between $\pm 2 \mathrm{~mm}$ from the required position. At each of these axial positions, the 
Table 1 Design and PET performance specifications

\begin{tabular}{ll}
\hline & PET \\
\hline Axial FOV & $25 \mathrm{~cm}$ \\
Transaxial FOV & $60 \mathrm{~cm}$ \\
Photodetector & SiPM \\
Scintillator & LYSO \\
Crystal element size & $25 \times 4.0 \times 5.3 \mathrm{~mm}^{3}$ \\
Electronics & Integrated in-bore \\
Time resolution & $<400 \mathrm{ps}$ \\
Energy window & $425-650 \mathrm{keV}$ \\
Coincidence timing window & $4.57( \pm 2.29 \mathrm{~ns})$ \\
NEMA NU 2-2007 PET test ${ }^{\mathrm{a}}$ & \\
Sensitivity & $22.5 \mathrm{cps} / \mathrm{kBq}$ \\
Peak NECR & $212.2 \mathrm{kcps}$ \\
$\quad$ Activity at Peak NECR & $18.1 \mathrm{kBq} / \mathrm{ml}$ \\
Scatter Fraction at Peak NECR & $44.1 \%$ \\
Spatial Resolution (Axial) & $(5.53-6.95) \mathrm{mm}$ at 1 and $10 \mathrm{~cm}$ \\
\hline
\end{tabular}

${ }^{\mathrm{a} G E}$ healthcare acceptance tests [23]

resolution was measured centrally in the FOV $(1 \mathrm{~cm}$ horizontal offset relative to the center) as well as $10 \mathrm{~cm}$ horizontal offset and $10 \mathrm{~cm}$ vertical offset relative to the center. Data was reconstructed with filtered back-projection. The full width at half maximum (FWHM) and full width at tenth of maximum (FWTM) of the point source response function in all three directions were determined by one-dimensional response functions along profiles though the image volume in three orthogonal directions.

\section{Sensitivity}

Sensitivity was tested with the NEMA sensitivity phantom, composed of a line source with 5 different thicknesses of aluminum. The 70-cm-long line source was filled with a volume of approximately $2.3 \mathrm{ml}$. The activity level was equal to $10.7 \mathrm{MBq}$ and $8.7 \mathrm{MBq}$ at scan start for ${ }^{18} \mathrm{~F}$ and ${ }^{68} \mathrm{Ga}$. For ${ }^{90} \mathrm{Y}$, the activity level was adjusted to $444.9 \mathrm{MBq}$, to compensate for the low positron abundance and to keep the scan time acceptable. Using this activity, the total number of counts collected was above 2 million counts. This measurement was done in the center of the FOV and at $10 \mathrm{~cm}$ away from the center of the FOV. Data was collected for a period of time to ensure that at least 10.000 trues per slice were collected. The system sensitivity was calculated by fitting the decay-corrected count rate of each acquisition to an exponential and extrapolating the value for a hypothetical acquisition with no aluminum tubes over the source (no attenuation). Axial sensitivity profiles were generated by calculating the sensitivity of each slice for the transaxially centered data acquisitions that used only the smallest aluminum tube.

\section{Scatter fraction, noise equivalent count rate (NECR)}

A 70-cm-long plastic tube line source $(3.2 \mathrm{~mm}$ in inner diameter) was filled with a calibrated activity of $905 \mathrm{MBq}$ and $871 \mathrm{MBq}$ in a $5.0 \mathrm{ml}$ of solution for ${ }^{18} \mathrm{~F}$ and ${ }^{68} \mathrm{Ga}$, 
respectively. The line source was inserted $4.5 \mathrm{~cm}$ below the central axis of a 70-cmlong cylindrical polyethylene test phantom. The center of the NEMA scatter phantom was positioned at the FOV center and the data were acquired overnight. After twentynine frames of data were extracted from the list mode data, NEMA specifications were used to derive the trues, randoms, scatter, and NECR from the prompts dataset in each frame. The results were plotted as a function of effective activity concentration. In addition, the accuracy of count losses and randoms corrections was determined by extrapolating image results from low count rates.

\section{Image quality, accuracy of attenuation, and scatter corrections}

Image quality was measured by acquiring the NEMA image quality phantom. A 5-cmdiameter cylindrical insert filled with Styrofoam pellets was positioned in the center of the phantom to simulate lung tissue. The warm background volume of the phantom was filled with an activity concentration of $5.3 \mathrm{kBq} / \mathrm{ml}$. Four hot spheres with diameters of 10, 13, 17, and $22 \mathrm{~mm}$ were filled with an activity concentration 4 times the background for ${ }^{18} \mathrm{~F}$ and ${ }^{68} \mathrm{Ga}$. For ${ }^{90} \mathrm{Y}$, a higher 8:1 ratio was used since typical contrasts in liver therapies are normally higher than 4:1. The two cold spheres with diameters of 28 and $37 \mathrm{~mm}$ were filled with water (except for the ${ }^{90} \mathrm{Y}$ image quality test, in which all of the spheres were filled with an $8: 1$ ratio of activity).

Background activity from outside the scanner FOV was generated by a line source inserted into the same cylindrical phantom as used in the scatter fraction, count losses, and randoms measurement. It contains $116 \mathrm{MBq}$ solution of the isotope used in the measurement and was placed on the bed axially adjacent to the body phantom. The percentage contrast recovery for the hot and cold spheres and the background variability were calculated, as defined in the NEMA standard. The percentage contrast recovery (in an ideal case $=100 \%$ ) is determined for each hot sphere $j$ by

$$
Q_{S, j}=\frac{\left(C_{S, j} / C_{B, j}\right)-1}{\left(a_{S} / a_{B}\right)-1} \cdot 100[\%]
$$

Where $C_{S, j}$ is the average counts of regions of interest (ROIs) on the spheres. These are positioned in the transverse image slice that contains the centers of the spheres. $C_{B}$, ${ }_{j}$ represents the average counts in the background ROI. The terms $a_{S}$ and $a_{B}$ are activity concentration in the hot spheres and background, respectively.

The phantom has also 2 large spheres which are not filled with isotope. For each nonradioactive sphere $j$, the percentage contrast recovery $Q_{C, j}$ was calculated by

$$
Q_{C, j}=\left(1-\frac{C_{C, j}}{C_{B, j}}\right) \cdot 100[\%]
$$

Where $C_{C, j}$ and $C_{B, j}$ are average counts in the ROI for sphere $j$ and average of all background ROI counts for sphere $j$.

In order to determine the percentage background variability $N_{j}$ as a measure for the image noise for sphere $j$ (in an ideal case $=0 \%$ ), the following equation was used:

$$
N_{j}=\left(\frac{S D_{j}}{C_{B, j}}\right) \cdot 100[\%]
$$

$S D_{j}$ is the standard deviation of the background ROI counts for sphere $j$. 
In addition, the central cylinder of the phantom did not contain any activity, and the relative error was calculated to determine the accuracy of scatter and attenuation correction as follows:

$$
\Delta C_{\text {lung }, i}=\frac{C_{\text {lung }, i}}{C_{B, i}} \cdot 100[\%]
$$

Where $\Delta C_{\text {lung, } i}$ is the relative error per percentage units for each slice $i, C_{\text {lung, } i}$ is the average counts in the lung insert ROI, and $C_{B, i}$ is the average of the $60(37 \mathrm{~mm})$ background ROIs drawn for the image quality analysis [19].

\section{Image-quality phantom images}

The phantom data were obtained with a 10 -min scan for ${ }^{18} \mathrm{~F}$ and ${ }^{68} \mathrm{Ga}$ acquisitions. For ${ }^{90} \mathrm{Y}$, a long 15-h scan time and a shorter $30 \mathrm{~min}$ representing a clinical acquisition were obtained using list mode data selection. The image quality phantom was reconstructed in a volume of 89 images using ordered subset expectation maximization reconstruction algorithm (OSEM) including time-of-flight (TOF) and scatter corrections with 2, 3, and 4 iterations of 28 subsets. The scatter correction for ${ }^{68} \mathrm{Ga}$ and ${ }^{90} \mathrm{Y}$ were defined as dirty emitters to account for the gamma. These isotopes allow an additional fitting parameter in the scatter tail scaling process [22]. All reconstruction schemes were performed using a matrix size of $256 \times$ 256 with $2.08 \times 2.08 \times 2.78-\mathrm{mm}^{3}$ voxel size, with $2 \mathrm{~mm}$ and no post-smoothing with and without point spread function (PSF). The GE PET/MR uses a system-generated approach that includes a CT-based template attenuation correction for the NEMA IQ phantom.

\section{Results}

\section{Spatial resolution}

The spatial resolution (FBP reconstruction) results for each isotope are presented in Table 2 . As a double check, the ${ }^{18} \mathrm{~F}$-measured values (at the same equipment across all isotopes) were used as a reference for all measurements performed on the GE Signa PET/MR. The FWHM radial and tangential resolution is slightly degraded for ${ }^{68} \mathrm{Ga}$ and ${ }^{90} \mathrm{Y}$. In comparison with ${ }^{18} \mathrm{~F}$-measured values, the relative differences were $17.8 \%$ and $-1.3 \%$ at $1 \mathrm{~cm}$ and $27.9 \%$ and $3.5 \%$ at $10 \mathrm{~cm}$ in the axial direction for ${ }^{68} \mathrm{Ga}$ and ${ }^{90} \mathrm{Y}$, respectively. With regards to the FWTM in the axial resolution at 1 and $10 \mathrm{~cm}$ off-center, the percentage difference relative to ${ }^{18} \mathrm{~F}$ values were $70 \%$, and $3.3 \%$ at $1 \mathrm{~cm}$ and $57.3 \%$ and $12.3 \%$ at $10 \mathrm{~cm}$ off-center for ${ }^{68} \mathrm{Ga}$ and ${ }^{90} \mathrm{Y}$, respectively.

\section{Sensitivity}

Sensitivity test results for ${ }^{18} \mathrm{~F},{ }^{68} \mathrm{Ga}$, and ${ }^{90} \mathrm{Y}$ are $21.8,20.1$, and $0.653 \cdot 10^{-3} \mathrm{cps} / \mathrm{kBq}$ at the center position and 21.2, 19.7, and $0.667 \cdot 10^{-3} \mathrm{cps} / \mathrm{kBq}$ at $10 \mathrm{~cm}$ off-center. Table 3 gives the measured average sensitivity values at the transaxial center and $10 \mathrm{~cm}$ off-center. Also, data are compared to the average ${ }^{18} \mathrm{~F}$ measured and theoretical values. These estimated values were calculated based on the difference in branching ratio relative to the ${ }^{18} \mathrm{~F}$. The sensitivity values are in line with the lower positron fraction of the isotopes.

\section{Scatter fraction, noise equivalent count rate (NECR)}

The peak NECR, the corresponding activity concentration and scatter fraction at peak NECR, is presented in Table 4 and Fig. 1 for ${ }^{18} \mathrm{~F}$ and ${ }^{68} \mathrm{Ga}$. Table 4 summarizes the 
Table 2 Spatial resolution tests

\begin{tabular}{llll}
\hline & ${ }^{18} \mathrm{~F}$ & ${ }^{68} \mathrm{Ga}$ & \\
\hline FWHM $(\mathrm{mm})$ & & & \\
At $1 \mathrm{~cm}$ & & & \\
$\quad$ Transverse ${ }^{\mathrm{a}}$ & 4.05 & 4.18 & 6.42 \\
$\quad$ Axial & 6.08 & 7.16 & \\
At $10 \mathrm{~cm}$ & & & 5.79 \\
$\quad$ Radial & 5.75 & 5.80 & 4.44 \\
Tangential & 4.38 & 4.6 & 7.09 \\
Axial & 6.85 & 8.76 & \\
FWTM (mm) & & & 9.02 \\
At 1 cm & & & 12.36 \\
Transverse & & 8.68 & \\
Axial & 8.62 & 20.35 & 10.78 \\
At $10 \mathrm{~cm}$ & 11.97 & & 8.84 \\
Radial & & 10.70 & 15.73 \\
Tangential & 10.68 & 8.78 & \\
Axial & 8.61 & 22.04 &
\end{tabular}

comparison between ${ }^{18} \mathrm{~F}$ and ${ }^{68} \mathrm{Ga}$ in terms of scatter fraction at peak NECR, peak NECR, activity concentration at peak NECR, and maximum absolute error.

For ${ }^{18} \mathrm{~F}$, the NECR has a maximum of $216.8 \mathrm{kcps}$ at an activity concentration of 18.6 $\mathrm{kBq} / \mathrm{ml}$. At the peak, the scatter fraction was $43.3 \%$, comparable to the results obtained on three separate scanners installed in three institutions [23].

For ${ }^{68} \mathrm{Ga}$, the trues, random, and scatter count rates at the same activity concentration are lower in comparison to ${ }^{18} \mathrm{~F}$. The measured NECR peak for ${ }^{68} \mathrm{Ga}$ was also clearly lower and peaks at $205.6 \mathrm{kcps}$. This peak is obtained at a higher activity concentration of $20.4 \mathrm{kBq} / \mathrm{ml}$. The scatter fraction at peak NECR was below $1 \%$ lower compared to ${ }^{18} \mathrm{~F}$ measured in our institution (Fig. 1a and Table 4). After the full 15-h acquisitions (for both isotopes), the maximum absolute value of the slice error was $3.0 \%$ and $6.0 \%$ for ${ }^{18} \mathrm{~F}$ and ${ }^{68} \mathrm{Ga}$.

Image quality, accuracy of attenuation, and scatter corrections

The results for contrast recovery versus sphere diameter of the image quality phantom are shown in Fig. 2. In Fig. 2 a, the contrast recovery of the reconstructed image of the phantom without PSF and post-smooth filter was lower for ${ }^{68} \mathrm{Ga}$ and ${ }^{90} \mathrm{Y}$ as shown in

Table 3 Sensitivity test results: comparison between the average sensitivity measured and theoretical values relative to ${ }^{18} \mathrm{~F}$

\begin{tabular}{llll}
\hline Isotope & Branching ratio & Average sensitivity measured $(\mathrm{cps} / \mathrm{kBq})$ & Theoretical values $(\mathrm{cps} / \mathrm{kBq})^{a}$ \\
\hline${ }^{18} \mathrm{~F}$ & 0.968 & 21.5 & - \\
${ }^{68} \mathrm{Ga}$ & 0.879 & 19.9 & 19.5 \\
${ }^{90} \mathrm{Y}$ & $31.86 \cdot 10^{-6}$ & $0.66 \cdot 10^{-3}$ & $0.71 \cdot 10^{-3}$ \\
\hline
\end{tabular}

aalues relative to ${ }^{18} \mathrm{~F}$-measured value 
Table 4 Scatter Fraction, peak NECR, and source activity test results for ${ }^{18} \mathrm{~F}$ and ${ }^{68} \mathrm{Ga}$

\begin{tabular}{llll}
\hline Scan type & Unit & Measured values & \\
\hline Isotopes & & ${ }^{18} \mathrm{~F}$ & ${ }^{68} \mathrm{Ga}$ \\
Scatter fraction at peak NECR & $\%$ & 43.3 & 42.9 \\
Peak NECR & $\mathrm{kcps}$ & 216.8 & 205.6 \\
Activity at peak NECR & $\mathrm{kBq} / \mathrm{ml}$ & 18.60 & 20.40 \\
Maximum absolute error & $\%$ & 3.0 & 6.0 \\
\hline
\end{tabular}

the relative difference to ${ }^{18} \mathrm{~F}$-measured values. The contrast recovery increased when compared using TOF, PSF, and post-smooth filter, as showed in Fig. 2 b.

The background variability and the lung region relative error for ${ }^{18} \mathrm{~F},{ }^{68} \mathrm{Ga}$, and ${ }^{90} \mathrm{Y}$ are shown in Table 5 for different sphere sizes. ${ }^{90} \mathrm{Y}$ has a much lower quality and suffers from low counts, for most spheres the background variability. The lung error is clearly higher than for ${ }^{18} \mathrm{~F}$ and ${ }^{68} \mathrm{Ga}$. This is also visually confirmed by the clinical reconstructions (30 min acquisition) in Fig. 3.

When compared in terms of number of iterations (Fig. 3), the noise level increased with increasing of the number of iterations for all isotopes.

\section{Discussion}

The aim of this study was to assess the impact of using different PET isotopes for the NEMA tests performance evaluation of the GE Signa integrated PET/MR. The performance and characteristics of PET/MR have been investigated based on NEMA NU 2-2012 with regard to ${ }^{18} \mathrm{~F}$ [20, 21, 23], but not for different PET isotopes. Furthermore, the NEMA NU 22012 version is only slightly different from the 2007 version; the most substantial changes are relatively minor, mostly designed to make the test easier to conduct, mere reproducible, or more clearly defined [19]. In this study, we conveniently choose to evaluate the PET/MR using NEMA NU 2-2007 because GE Healthcare has reported their acceptance testing on this version [24].

The system performance of the GE Signa integrated PET/MR was substantially different, in terms of NEMA spatial resolution and image quality for ${ }^{68} \mathrm{Ga}$ and ${ }^{90} \mathrm{Y}$ PET imaging test as compared to ${ }^{18} \mathrm{~F}$. In the transverse plane, the magnetic field reduces the effective positron range, and the dominant factor on spatial resolution seems to be the detector pixel size and the transverse resolution is therefore comparable with the result

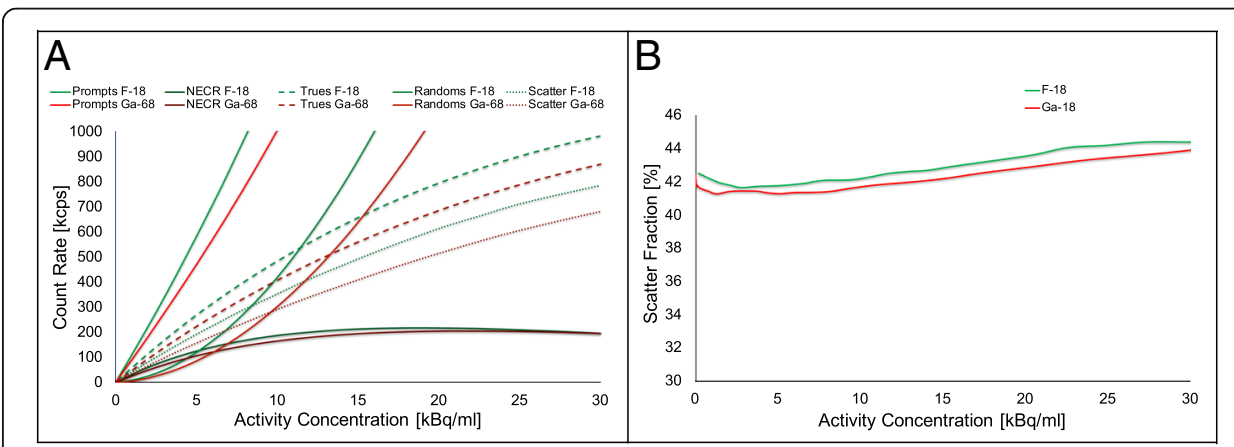

Fig. 1 NEMA counting rate measurements: count rates (a) and scatter fraction (b) vs activity concentration for the both isotopes ${ }^{18} \mathrm{~F}$ and ${ }^{68} \mathrm{Ga}$. Notice than peak of the NECR curve (a) of ${ }^{68} \mathrm{Ga}$ is lower (at clinical NECR) and appears at higher activity concentrations 


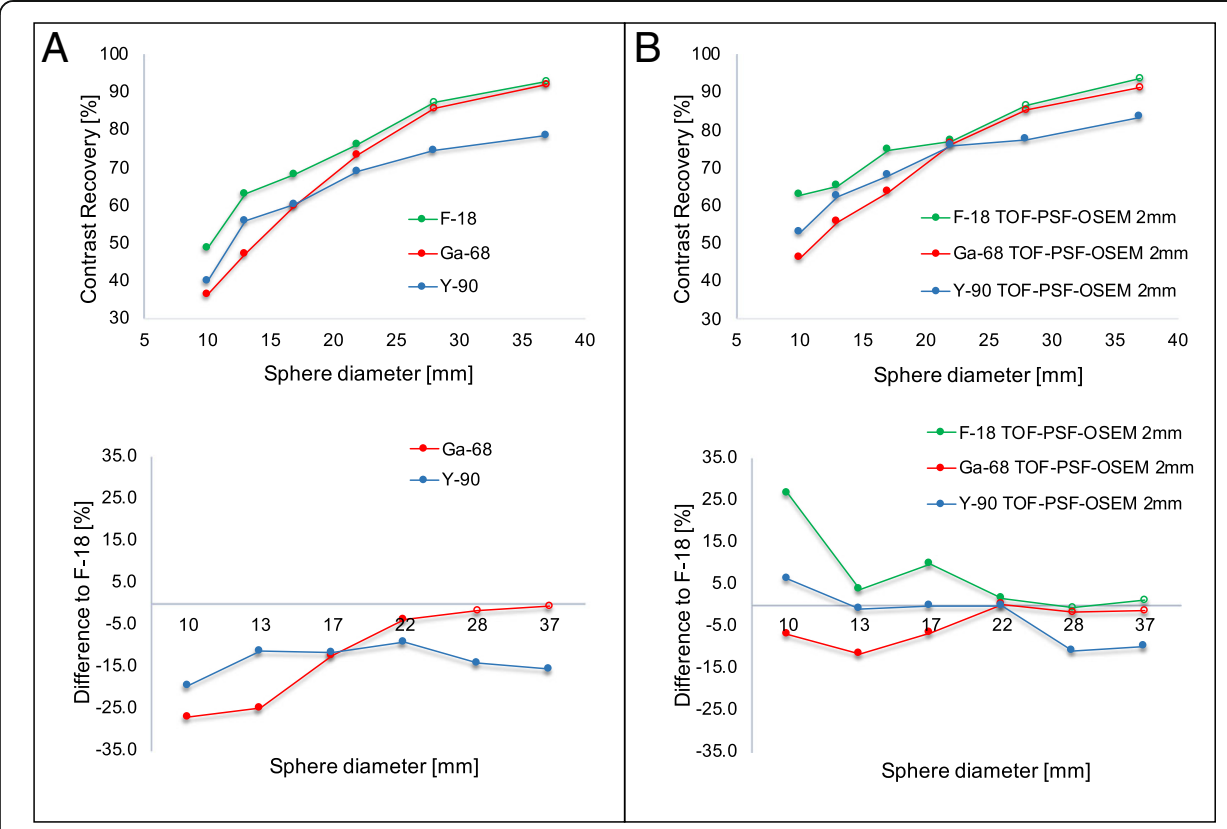

Fig. 2 Contrast recovery of TOF-OSEM 4 iteration and 28 subsets as a function of sphere size for $10 \mathrm{~min}\left({ }^{18} \mathrm{~F}\right.$ and ${ }^{68} \mathrm{Ga}$ ) and $15 \mathrm{~h}\left({ }^{90} \mathrm{Y}\right)$ acquisition times and different isotopes. The cold and radioactive spheres are indicate using open $\left({ }^{18} \mathrm{~F}\right.$ and $\left.{ }^{68} \mathrm{Ga}\right)$ and filled circles $\left({ }^{90} \mathrm{Y}\right)$, respectively. Contrast recovery and percentage difference relative to ${ }^{18} \mathrm{~F}$ for each sphere size: $\mathbf{a}$ without PSF and post-smoothing filter and $\mathbf{b}$ with PSF and $2 \mathrm{~mm}$ post-smoothing filter

of ${ }^{18} \mathrm{~F}$. This effect was confirmed by other studies with simulations for different isotopes and field strengths $[25,26]$. The main magnetic field along the axial direction leads to an increased positron range in this direction and a pronounced reduction of the range in the transversal plane for high-energy positrons. On the other hand, the positron range effect of the magnetic field is not significant for ${ }^{18} \mathrm{~F}$ [27] and no significative effect was found for ${ }^{90} \mathrm{Y}$. In agreement with these studies, the FWHM difference relative to ${ }^{18} \mathrm{~F}$-measured values were $17.8 \%$ and $-1.3 \%$ at $1 \mathrm{~cm}$ and $27.9 \%$ and $3.5 \%$ at $10 \mathrm{~cm}$ off-center in the axial direction for ${ }^{68} \mathrm{Ga}$ and ${ }^{90} \mathrm{Y}$, respectively, as shown in Table 2. However, the NEMA spatial resolution test is designed to characterize the detector, rather than the isotope which leads to limitations to account the effect of magnetic field on positron range on the transaxial resolution measurements. The capillary

Table 5 Background variability and lung error of TOF-OSEM 4 iteration and 28 subsets image reconstructed, with and without PSF and $2 \mathrm{~mm}$ post-smooth filter for ${ }^{18} \mathrm{~F}$, ${ }^{68} \mathrm{Ga}$, and ${ }^{90} \mathrm{Y}$ respectively

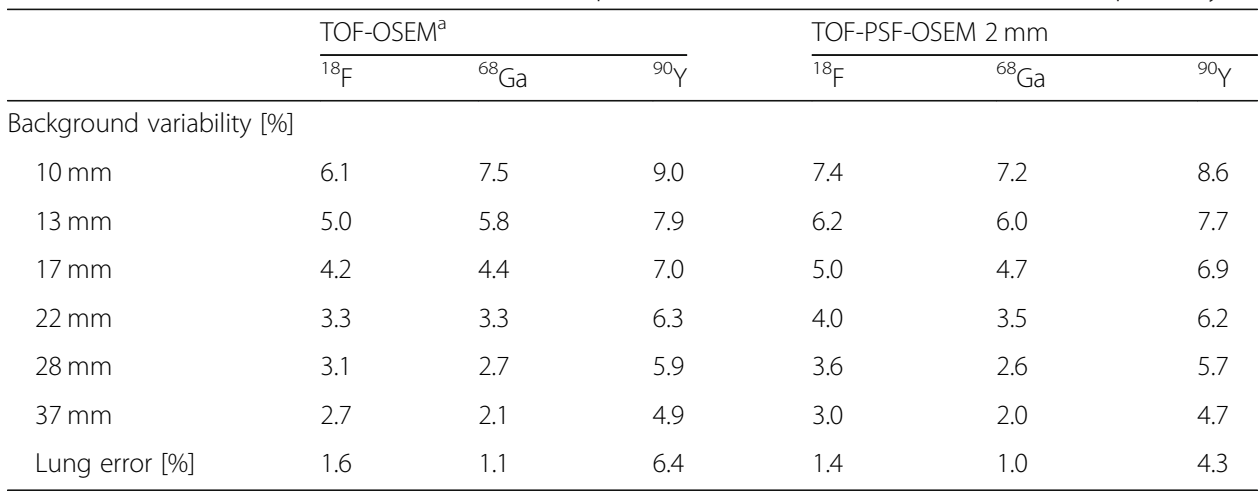

${ }^{\mathrm{a}} 4$ iterations and 28 subsets 


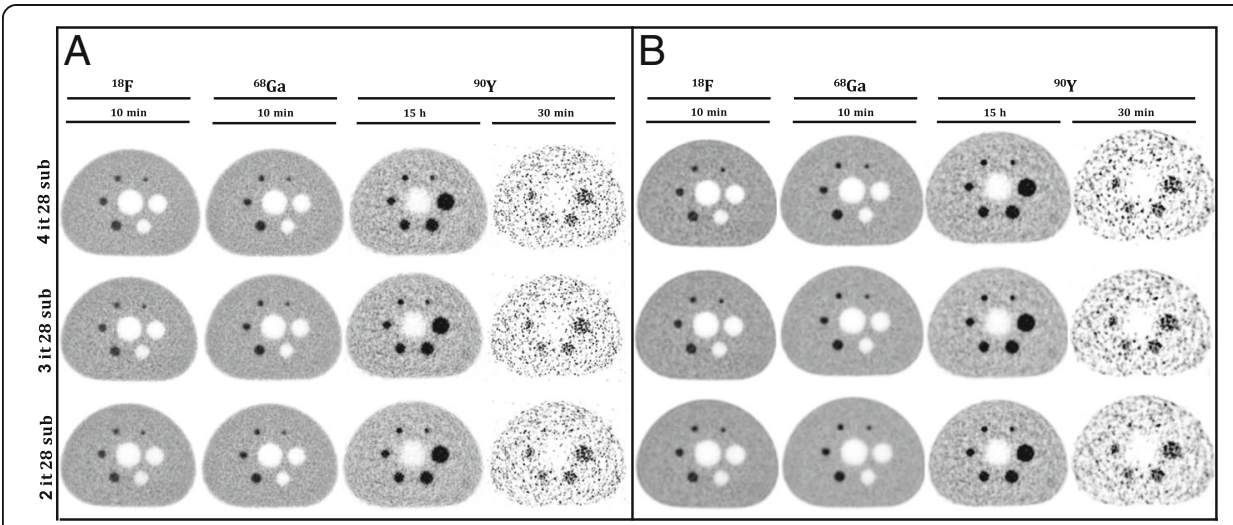

Fig. 3 Axial slices of the phantom reconstructed using TOF-OSEM for 2, 3, and 4 iterations and 28 subsets for different acquisition times and different isotopes: $\mathbf{a}$ without PSF and post-smoothing filter and $\mathbf{b}$ with PSF and $2 \mathrm{~mm}$ Gaussian filter

is very small, and any positron that escapes the capillary is not accounted for in the measurements. In addition, axially, the annihilation could occur in the tube-sealing compound and beyond that, the axial test is slightly poor due to the rebinning process and larger pixel size in the $z$-axis.

The NEMA image quality test was also substantially different in the measured contrast recovery, as shown in Fig. 2. It seems that the inferior resolution also affects the contrast recovery of the radioactive spheres in the NEMA quality phantom for ${ }^{68} \mathrm{Ga}$ and ${ }^{90} \mathrm{Y}$. While the results look visually (Fig. 3) similar between ${ }^{18} \mathrm{~F}$ and ${ }^{68} \mathrm{Ga}$ for TOFOSEM without resolution modeling and post-smooth filter, there is (Fig. 2a) a clearly lower contrast recovery for the smaller spheres in ${ }^{68} \mathrm{Ga}$ and also lower contrast recovery in ${ }^{90} \mathrm{Y}$, which is probably caused by the increased positron range and loss in resolution. A similar approach using different PET isotopes in a brain phantom measured at different field strengths was conducted by Shah et al. [27]. The contrast of the reconstructed image of the brain phantom filled with ${ }^{68} \mathrm{Ga}$ was significantly affected by the magnetic field in the axial direction more than ${ }^{18} \mathrm{~F}$ (low-energy positron emitter). However, errors in scatter correction and the use of different sphere to background ratios might have influenced these results [3, 23, 28, 29]. A low-frequency offset of the data makes the images appear to have more or less contrast recovery. And different ratios lead to different contrast recovery. This can explain the crossing of the ${ }^{90} \mathrm{Y}$ curve (ratio 8:1) in Fig. 2.

With regards to noise level and the average lung residual error (Table 5), the results were comparable between ${ }^{18} \mathrm{~F}$ and ${ }^{68} \mathrm{Ga}$, but for ${ }^{90} \mathrm{Y}$, the background variability and the lung error are clearly higher than for ${ }^{18} \mathrm{~F}$ and ${ }^{68} \mathrm{Ga}$, which is also visually seen $(15 \mathrm{~h}$ and 30 min acquisition) in Fig. 3. However, when comparing the reconstructed images using resolution modeling and $2 \mathrm{~mm}$ post-smooth Gaussian filter to reduce the noise, the contrast recovery increased with acceptable noise level, as shown in Figs. 2b and 3b. Although high-energy positron emitters are affected by the field strengths, recent developments in reconstruction methods including dedicated positron range correction have successfully corrected this effect [30]. A new Bayesian penalized likelihood reconstruction algorithm which uses a block sequential regularized expectation maximization as 
an optimizer (including TOF and PSF) was introduced in the last few years by GE Healthcare (Q.Clear) on their PET scanners in order to improve clinical image quality. Unlike traditional OSEM reconstruction, which increases the noise with the number of iterations (Fig. 3), this algorithm improves image quality by controlling noise amplification during image reconstruction [31].

The mean sensitivity results shown in Table 3 are in line with theoretical values as expected for ${ }^{68} \mathrm{Ga}$ and ${ }^{90} \mathrm{Y}$ test. The primary factor for the sensitivity change is the scale factor related to the positron emission fraction $\left(96.7 \%, 87.9 \%\right.$, and $0.003186 \%$ for ${ }^{18} \mathrm{~F}$, ${ }^{68} \mathrm{Ga}$, and ${ }^{90} \mathrm{Y}$, respectively). The low branching ratio of ${ }^{90} \mathrm{Y}$ explains the substantial quality difference of the reconstructed transverse image quality phantom as compared with ${ }^{18} \mathrm{~F}$ and ${ }^{68} \mathrm{Ga}$ images, as shown in Fig. 3. In several design factors (Table 2) including Compton scatter recovery [31, 32], the longer axial FOV and reduced detector ring diameter lead to higher count rates and an increased sensitivity, both in stand-alone operation and with simultaneous MR image acquisition [33].

NEMA count rate performance and accuracy measurements summarized in Table 4 and Fig. 1a suggest that the scanner provides excellent accurate quantitative measurements and utilizes effective randoms and dead time correction methods for ${ }^{18} \mathrm{~F}$ [23]. For ${ }^{68} \mathrm{Ga}$, the scatter fraction at NECR peak (Table 4 and Fig. 1b) was slightly lower compared to ${ }^{18} \mathrm{~F}$ measured in our institution and the GE test. This is primarily due to $1.2 \%(1.883 \mathrm{MeV})$ fraction of ${ }^{68} \mathrm{Ga}$ that decays by $\beta_{2}^{+}$can result in a small prompt gamma $(1.077 \mathrm{MeV})$ [12-14] contamination into the PET data. The prompt gammas of ${ }^{68} \mathrm{Ga}$ can directly fall into the energy window and accepted by the PET scanner. This happens when the $1.077 \mathrm{MeV}$ scatters in the phantom and generates an energy falling in the main energy window. In this case, there will be a coincidence with a true 511 $\mathrm{keV}$ resulting from the same decay [18]. Contributions in which only the gamma is detected would add to randoms which does not affect the calculation for scatter fraction.

The ${ }^{68} \mathrm{Ga}$ NECR test was clearly lower than measured for ${ }^{18} \mathrm{~F}$ and appears at a slightly higher activity concentration (Fig. 1a). The lower peak NECR can be explained by the additional $1.077 \mathrm{MeV}$ gamma which leads to additional detections increasing the deadtime of the detector blocks. These can also lead to additional randoms or scatter when they lose enough energy for falling into the energy window. However, the effect from these promptgammas is clearly very small from a scatter fraction perspective and concerning for all activity concentration values below NECR (Table 4), and the maximum absolute value of the slice error is $2.9 \%$ and $6.0 \%$ for ${ }^{18} \mathrm{~F}$ and ${ }^{68} \mathrm{Ga}$, respectively. There is also no appreciable impact in the measured residual activity of the lung insert in the IQ phantom (Table 5).

In summary, the overall GE Signa PET/MR system performance with TOF capability based on SiPM detectors, shows substantially different system characteristics for each of these commercially available isotopes. However, the NEMA spatial resolution test is designed to characterize the detector, rather than the isotope, which needs to be adapted in order to well account for the effect of magnetic field on positron range on the transaxial resolution measurements. The variety of prompt gammas in coincidences of these isotopes and the interference of the MR field on positron range, however, seem to have been compensated by the PET scanner technologies, which, in combination with recently developments in reconstruction methods (regularized TOF OSEM and PSF), lead to a comparable noise equivalent count rate and a good scatter fraction. 


\title{
Conclusions
}

NEMA NU 2-2007 performance measurements using ${ }^{18} \mathrm{~F},{ }^{68} \mathrm{Ga}$, and ${ }^{90} \mathrm{Y}$ resulted in substantially different system characteristics, specifically in terms of spatial resolution and recovery coefficients of the image quality measurements. NEMA spatial resolution test needs to be adapted in order to correctly account for the difference in positron range in the transaxial resolution measurements. And when NEMA image quality test is compared using TOF-OSEM-PSF and post-smooth gaussian filter, the contrast recovery increased with acceptable noise level. The primary factor for the sensitivity change can be explained by the scale factor related to the positron emission fraction of the isotopes. Scatter fraction and NECR differences between the ${ }^{18} \mathrm{~F}$ and ${ }^{68} \mathrm{Ga}$ are relatively small: for ${ }^{68} \mathrm{Ga}$, the peak NECR is lower and appears at higher activity concentrations. The maximum absolute value of the slice error is $2.9 \%$ and $6.0 \%$ for ${ }^{18} \mathrm{~F}$ and ${ }^{68} \mathrm{Ga}$, respectively. These performance results are compensated by the PET scanner technologies and reconstructions methods.

\begin{abstract}
Abbreviations
CT: Computed tomography; DTPA: Diethylenetriaminepentaacetic acid; FOV: Field of view; GE: General Electric Company; LBS: Lutetium-based scintillator; MR: Magnetic resonance; NECR: Noise equivalent count rate; NEMA: National Electrical Manufacturers Association; OSEM: Ordered subset expectation maximization; PET: Positron emission tomography; PSF: Point spread function; PSMA: Prostate membrane antigen; RC: Recovery coefficient; ROI: Region of interest; SiPM: Silicon photomultipliers; SUV: Standardized uptake value; TOF: Time of flight; VOI: Volume of interest
\end{abstract}

Acknowledgments

Not applicable

Disclosure

T. W. Deller is an employee of GE Healthcare. No other potential conflict of interest relevant to this article was reported.

Authors' contributions

All authors contributed to design of the study. PC and MK performed data collection and analysis. All authors discussed the results and implications and commented on the manuscript. All authors read and approved the final manuscript.

Funding

This present study was conducted with a grant support to the first author from CNPq, the National Council of Technological and Scientific Development - Brazil (process number 235040/2014-2).

\section{Availability of data and materials}

The datasets used and/or analyzed during the current study are available from the corresponding author on reasonable request.

Ethics approval and consent to participate

Not applicable

Consent for publication

Not applicable

Competing interests

The authors declare that they have no competing interests.

\section{Author details}

${ }^{1}$ Medical Imaging and Signal Processing - MEDISIP, Ghent University, Corneel Heymanslaan 10, 9000 Ghent, Belgium. ${ }^{2}$ Division of Nuclear Medicine and Molecular Imaging, UZ/KU Leuven, Herestraat 49 B-3000, Leuven, Belgium. ${ }^{3} \mathrm{GE}$ Healthcare, Waukesha, WI 53188-1678, USA.

Received: 3 February 2019 Accepted: 14 June 2019

Published online: 04 July 2019

\section{References}

1. Vandenberghe S, Marsden PK. PET-MRI: a review of challenges and solutions in the development of integrated multimodality imaging. Phys Med Biol. 2015;60(4):R115-54.

2. Yamamoto S, Watabe H, Kanai Y, Aoki M, Sugiyama E, Watabe T, Imaizumi M, Shimosegawa E, Hatazawa J. FPGA-based RF interference between PET and MRI sub-systems in a silicon-photomultiplier-based PET/MRI system. Phys Med Biol. 2011;56:4147-59. https://doi.org/10.1088/0031-9155/61/9/3500. 
3. Levin CS, Maramraju SH, Khalighi MM, Deller TW, Delso G, Jansen F. Design features and mutual compatibility studies of the time-of-flight PET capable GE SIGNA PET/MR system. IEEE Trans Med Imaging. 2016;35(8):1907-14.

4. Delso G, Ziegler S. PET/MRI system design. Eur J Nucl Med Mol Imag. 2009;36 Suppl 1:S86-92. https://doi.org/10.1007/ s00259-008-1008-6.

5. Bai B, Li Q, Leahy RM. Magnetic resonance-guided positron emission tomography image reconstruction. Semin Nucl Med. 2013;43(1):30-44.

6. Judenhofer MS, Cherry SR. Applications for preclinical PET/MRI. Semin Nucl Med. 2013;43:19-29. https://doi.org/10.1053/ j.semnuclmed.2012.08.004.

7. Conti M, Eriksson L. Physics of pure and non-pure positron emitters for PET: a review and a discussion. EJNMMI Phys. 2016;3(1):8. https://doi.org/10.1186/s40658-016-0144-5.

8. Banerjee SR, Pomper MG. Clinical applications of Gallium-68. Appl Radiat Isot. 2013;76:2-13. https://doi.org/10.1016/j. apradiso.2013.01.039.

9. Afshar-Oromieh A, Zechmann CM, Malcher A, Eder M, Eisenhut M, Linhart HG, et al. Comparison of PET imaging with a ${ }^{68} \mathrm{Ga}$-labelled PSMA ligand and ${ }^{18} \mathrm{~F}$-choline-based PET/CT for the diagnosis of recurrent prostate cancer. Eur J Nucl Med Mol Imaging. 2014;41(1):11-20. https://doi.org/10.1007/s00259-013-2525-5.

10. Freitag MT, Radtke JP, Hadaschik BA, et al. Comparison of hybrid ${ }^{68}$ Ga-PSMA PET/MRI and ${ }^{68} \mathrm{Ga}-\mathrm{PSMA}$ PET/CT in the evaluation of lymph node and bone metastases of prostate cancer. Eur J Nucl Med Mol Imaging. 2016;43:70. https://doi. org/10.1007/s00259-015-3206-3.

11. Schmin DT, John H, Zweifel R, Cservenyak T, Westera G, Goerres GW, et al. Fluorocholine PET/CT in patients with prostate cancer: initial experience. Radiology. 2005;235:623-8. https://doi.org/10.1148/radiol.2352040494.

12. Andreyev A, Celler A. Dual-isotope PET using positron-gamma emitters. Phys Med and Biol. 2011;56:453956. https://doi. org/10.1088/0031-9155/56/14/020

13. Cal-González J, Moore SC, Park MA, et al. Improved quantification for local regions of interest in preclinical PET imaging. Phys Med Biol. 2015;60(18):7127-49.

14. Velikyan I. Prospective of ${ }^{68} \mathrm{Ga}$-radiophamaceutical developments. Theranostics. 2014;4:47-80. https://doi. org/10.7150/thno.7447.

15. Pandey U, Mukherjee A, Sarma HD, Das T, Pillai MRA, Venkatesh M. Evaluation of ${ }^{90}$ Y-DTPA and ${ }^{90}$ Y-DOTA for potential application in intra-vascular radionuclide therapy. Appl Radiat Isot. 2002;57(3):313-8.

16. Carlier T, Willowson KP, Fourkal E, Bailey DL, Doss M, Conti M. ${ }^{90} \mathrm{Y}$-PET imaging: exploring limitations and accuracy under conditions of low counts and high random fraction. Med Phys. 2015;42:4295. https://doi.org/10.1118/1.4922685.

17. Rao AV, Akabani G, Rizzieri DA. Radioimmunotherapy for non-Hodgkin's lymphoma. Clin Med Res. 2005;3(3):157-65.

18. Lhommel R, Van Elmbt L, Goffette P, et al. Feasibility of ${ }^{90} \mathrm{Y}$ TOF PET-based dosimetry in the liver metastasis therapy using SIR-spheres. Eur J Nucl Med Mol Imaging. 2010;37(9):1654-62.

19. National Electrical Manufacturers Association. NEMA Standards Publication NU 2-2007, Performance measurements of positron emission tomographs: Rosslyn; 2007. p. 26-33. https://www.nema.org. Accessed 14 Dec 2018

20. Ronald B, Ivo R, Thomas B, Gaspar D, Maqsood Y, Harald HQ, Bernhard S. Quality control for quantitative multicenter whole-body PET/MR studies: a NEMA image quality phantom study with three current PET/MR systems. Med Phys. 2015; 42:5961. https://doi.org/10.1118/1.4930962.

21. Susanne Z, Bjoern WJ, Harald B, Daniel HP, Harald HQ. NEMA image quality phantom measurements and attenuation correction in integrated PET/MR hybrid imaging. Ziegler et al ENNMMI Physics. 2015;2:18. https://doi.org/10.1186/s40658-015-0122-3.

22. Braad PEN, et al. PET imaging with the non-pure positron emitters: ${ }^{55} \mathrm{Co}$, ${ }^{86} \mathrm{Y}$ and ${ }^{124}$. Phys Med Biol. 2015;60(9):3479

23. Grant AM, Deller TW, Khalighi MM, Maramraju SH, Delso G, Levin CS. NEMA NU 2-2012 performance studies for the SiPMbased ToF-PET component of the GE SIGNA PET/MR system. Med Phys. 2016;43:2334-43. https://doi.org/10.1118/1.4945416.

24. GE Healthcare: SIGNA PET/MR NEMA NU 2-2007 Manual. (2007). https:/www.nema.org/Manufacturers/Pages/GEHealthcare.aspx Accessed 11 Dec 2018.

25. Kraus R, Delso G, Ziegler SI. Simulation study of tissue-specific positron range correction for the new biograph mMR IEEE Trans Nucl Sci. 2012;59(5):1900-9. https://doi.org/10.1109/TNS.2012.2207436.

26. Shih-ying $H$, Dragana $S$, Jaewon $Y$, Uttam S, Youngho $S$. The effect of magnetic field on positron range and spatial resolution in an integrated whole-body time-of-flight PET/MRI system. IEEE Nucl Sci Symp Conf Rec Nov. 2014. https:// doi.org/10.1109/NSSMIC.2014.7431006

27. Jon Shah N, Hans H, Christoph W, Lutz T, Joachim K, Liliana C, Elena RK, Syed MQ, Heinz HC, Hidehiro I. Effects of magnetic fields of up to $9.4 T$ on resolution and contrast of PET images as measured with an MRBrainPET. PLOS One. 2014;9(4):e95250. https://doi.org/10.1371/journal.pone.0095250.

28. Spasic E, Jehanno N, Bblondeel-Gomes S, Huchet V, Luporsi M, Mounat TC. Phantom and clinical evaluation for new PET/CT reconstruction algorithm: Bayesian penalized likelihood reconstruction algorithm Q.Clear. J Nucl Med Radiat Ther. 2018;9(4):371. https://doi.org/10.4172/2155-9619.1000371.

29. Deller Timothy W, Khalighi MM, Jansen FP, Glover GH. PET imaging stability measurements during simultaneous pulsing of aggressive MR sequences on the SIGNA PET/MR system. J Nucl Med. 2018;59(1):167-72.

30. Ottavia BB, Afroditi E, Matteo C, Niccolò C, Nicola B, Charalampos T. PET iterative reconstruction incorporating an efficient positron range correction method. Physica Medica. 2016;32:323-30. https://doi.org/10.1016/j.ejmp.2015.11.005.

31. Hsu DFC, llan E, Peterson WT. Studies of a next-generation silicon-photomultiplier-based time-of-flight PET/CT system. J Nucl Med. 2017;58(9):1511-8. https://doi.org/10.2967/jnumed.117.189514.

32. Wagadarikar AA, Adrian I, Sergei D, MCDaniel DL. Sensitivity improvement of time-of-flight (ToF) PET detector through recovery of Compton scattered annihilation photons. IEEE Trans Nucl Sci. 2014;61(1):121-5.

33. Andrei I, et al. Simultaneous whole-body time-of-flight ${ }^{18} \mathrm{~F}$-FDG PET/MRI: a pilot study comparing SUVmax with PET/CT and assessment of MR image quality. Clin Nucl Med. 2015;40(1):1-8. https://doi.org/10.1097/RLU.0000000000000611.

\section{Publisher's Note}

Springer Nature remains neutral with regard to jurisdictional claims in published maps and institutional affiliations. 